(36) Gidley, M. J. Carbohydr. Res. 1987, 161, 301-304.

(37) Rees, D. A. J. Chem. Soc. B 1970, 877-884.

(38) Torsion angles $\phi$ and $\Psi$ are defined by $\mathrm{H}(1)-\mathrm{C}(1)-\mathrm{O}(1)-\mathrm{C}\left(4^{\prime}\right)$ and $\mathrm{H}\left(4^{\prime}\right)-\mathrm{C}\left(4^{\prime}\right)-\mathrm{O}(1)-\mathrm{C}(1)$, respectively, ${ }^{37}$ and are the major determinants of polymer conformational variations. ${ }^{20}$

(39) Imberty, A.; Chanzy, H.; Perez, S.; Buleon, A.; Tran, V. Macromolecules $1987,20,2634-2636$.

(40) Wu, H.-C. H.; Sarko, A. Carbohydr. Res. 1978, 61, 27-40.

(41) Morris, E. R.; Rees, D. A.; Young, G.; Walkinshaw, M. D.; Darke, A. J. Mol. Biol. 1977, 110, 1-16.
(42) Stipanovic, A. J.; Stevens, E. S. ACS Symp. Ser. 1981, No. 150, 303-315.

(43) Pfannemüller, B. Int. J. Biol. Macromol. 1987, 9, 105-108.

(44) Pangborn, W.; Langs, D.; Perez, S. Int. J. Biol. Macromol. 1985, 7, 363-369.

(45) Gidley, M. J.; Bociek, S. M. J. Am. Chem. Soc. 1988, 110, 3820-3829.

(46) Imberty, A.; Chanzy, H.; Perez, S.; Buleon, A.; Tran, V. J. Mol. Biol. 1988, 201, 365-378.

(47) Imberty, A.; Perez, S. Biopolymers 1988, 27, 1205-1221.

\title{
Rheology of Solutions of Rodlike Polymers: Theory and Comparison with Experiments
}

\author{
Burak Erman and Ivet Bahar \\ School of Engineering, Bogazici University, Bebek 80815, Istanbul, Turkey
}

\section{Patrick Navard*}

Centre de Mise en Forme des Materiaux, Ecole Nationale Superieure des Mines de Paris, Sophia Antipolis, 06560 Valbonne, France. Received October 23, 1987;

Revised Manuscript Received April 6, 1988

\begin{abstract}
The Doi theory (J. Polym. Sci., Polym. Phys. Ed. 1981, 19, 229) of concentrated solutions of rodlike particles is compared with the recent treatment of Bahar and Erman (J. Polym. Sci., Polym. Phys. $E d .1986,24,1361$ ) of the lattice theory of rods in a potential flow field. The Doi theory is modified by introducing a flow term to its effective mean-field potential, similar to that of the lattice treatment. Results of calculations based on the modified Doi theory are in agreement with existing viscosity concentration data on $\alpha$-helical poly(benzylglutamate) in $m$-cresol. At relatively low shear rates, the experimentally observed sharp maximum in viscosity is found to be located in the biphasic region. In this region the orientational order parameter and viscosity are double-valued. The characteristic features of the biphasic regime predicted by the theory are discussed. The viscosity-concentration curves exhibit smoother maxima at higher shear rates, although no phase separation is predicted by the theory. As the flow rate is further increased, the maximum gradually disappears in agreement with experiments. Also, the experimentally observed Newtonian plateau in the plots of viscosity against shear rates is obtained by the theory. Quantitative agreement between theory and experiment fails at high shear rates.
\end{abstract}

\section{Introduction}

Equilibrium statistics of solutions of rodlike particles have been formulated by Onsager, ${ }^{1}$ Isihara, ${ }^{2}$ and Flory. ${ }^{3}$ These theories show that a solution of rodlike polymers separates into an isotropic and an anisotropic phase above a critical concentration, $v^{*}$, depending on the axial ratio of the molecules. The presence of a critical concentration results solely from the effects of steric repulsions between the rodlike solute particles. It was also shown by theory ${ }^{3}$ that at a higher concentration $v_{2}{ }^{\mathrm{A}}$, the isotropic phase becomes unstable and the solution becomes completely anisotropic. The treatment of the problem according to lattice statistics by Flory has been improved ${ }^{4}$ where distribution of orientations of rods are calculated rigorously and the effects of thermotropic interactions ${ }^{5}$ are included. The basic predictions of the equilibrium theory lead to the following relations between $v^{*}, v_{2}{ }^{\mathrm{A}}$, and the axial ratio, $x$, of rods

$$
v^{*}=\frac{8}{x}(1-2 / x) \quad v_{2}{ }^{A}=11.6 / x
$$

which are in qualitative agreement with results of various experiments on solutions of $\alpha$-helical poly ( $\gamma$-benzyl-Lglutamate) in different solvents. ${ }^{6-11}$

The concentrations $v_{2}^{*}$ and $v_{2}{ }^{A}$ may be determined by steady flow viscosity measurements. Results of experiments in this field have critically been discussed by Matheson ${ }^{12}$ where it is deduced that the maximum in the viscosity-concentration diagram should lie between $v_{2}^{*}$ and $v_{2}{ }^{A}$.

The equilibrium lattice treatment of Flory ${ }^{3}$ has been modified by Marrucci and Ciferri ${ }^{13}$ by superposing a flow potential on the Gibbs free energy of the quiescent solution. Their analysis have recently been improved by Bahar and Erman ${ }^{14}$ by adopting the more recent lattice treatment $t^{4}$ of Flory and Ronca. It has thus been possible to extend the statistical theory for quiescent solutions to systems in steady-state potential flows for which the concentration dependence of flow-induced orientations and transitions may be calculated as a function of the imposed flow field.

Dynamics of solutions of rodlike molecules have been studied by Doi ${ }^{15}$ and Doi and Edwards. ${ }^{16}$ Uncrossability of the rods due to steric repulsions, which is the fundamental feature of the lattice treatment, has been incorporated into the kinetic equations governing the behavior of rods in concentrated solutions. The theory has later been extended by $\mathrm{Doi}^{17}$ to be valid at all concentrations. In addition to predicting the presence of $v^{*}{ }_{2}$ and $v_{2}{ }^{\mathrm{A}}$, the theory of Doi provides with a constitutive equation that relates the components of the stress tensor to the degree of orientation at vanishingly small shear rates. When solved numerically, the Doi equations may provide a description of the rheological behavior of rigid-rod lyotropic polymers. An attempt was recently made by Metzner and Prilutski ${ }^{18}$ where predictions of the Doi theory were com- 
pared with data from a $40 \%$ solution of (hydroxypropyl)cellulose (HPC) in acetic acid. The Doi theory was subsequently modified by Doraiswamy and Metzner ${ }^{19}$ by taking into account the dissipation effects of the solvent. This increases the number of adjustable parameters from three in their previous treatment ${ }^{18}$ to four and thus allows a finer fit with experimental data.

The Doi theory describes the dynamics of the rods and thus can provide information on viscosity, flow relaxations, etc... The lattice treatment on the other hand is derived from an equilibrium potential which has been extended to include a potential flow. In the present work, we attempt to identify the resemblances and differences between the Doi theory ${ }^{17}$ and the lattice theory ${ }^{14}$ of solutions of rodlike particles. The Doi theory is then used as a basis for relating the shear viscosity to the degree of ordering of the solution which is provided by the lattice theory. Results of the calculations are compared with two independent sets of experimental data on poly $(\gamma$-benzyl-Lglutamate) (PBLG), the works of Hermans ${ }^{6}$ and Kiss and Porter. ${ }^{22}$ The choice of PBLG is motivated by the fact that this system is known to be a very rigid helical polymer, and its rheology was investigated for several molecular weights. It is also one of the few mesomorphic polymers which does not show a power-law region at very low shear rates, which allows an easier comparison with theory. This does not mean that the PBLG solutions are monodomain nematics at low shear rates. Since, at the present time, no theory can easily predict the rheological behavior of a textured nematic polymer and there is no way to flow a defect-free nematic polymer, this comparison is the best that can be done.

\section{Lattice Treatment of Solutions of Rods in Potential Flow}

In this section, the free energy of a lyotropic solution of rods in steady-state potential flow is reviewed. The reader is referred to ref 4 for further details.

The change in free energy, $\Delta G$, due to mixing and flow of a system of rods in solution is given as

$$
\begin{aligned}
& \frac{\Delta G}{k T}=n_{1} \ln v_{1}+n_{2} \ln \left(\frac{v_{2}}{x}\right)-\left(n_{1}+\bar{y} n_{2}\right) \times \\
& \ln \left[1-v_{2}\left(1-\frac{\bar{y}}{x}\right)\right]+n_{2}(\bar{y}-1)-\sum_{y} n_{2 y} \ln \left(\frac{n_{2}}{n_{2 y}} \omega_{y}\right)+ \\
& n_{2} \ln \omega_{1}+n_{2} \frac{G}{2}\left(x^{3}+3 x^{2}+2 x\right)\left\langle\sin ^{2} \psi\right\rangle
\end{aligned}
$$

where $k$ is the Boltzmann constant, $T$ is the absolute temperature, $n_{1}$ and $n_{2}$ are the numbers of solvent and solute molecules, $v_{1}$ and $v_{2}$ are their respective volume fractions, and the angular brackets denote the ensemble average. $x$ is the axial ratio of rods which is equal to the number of isodiametric segments, each of a size equal to that of a lattice cell. The parameter $y$ is defined by

$$
y=(4 / \Pi) x \sin \psi
$$

where $\psi$ is the angle between the direction of alignment imposed by the flow field and the rod axis. Defined in this manner, $y$ is referred to as the disorientation index. The average $\bar{y}$ is defined as

$$
\bar{y}=\frac{4}{\Pi} x\langle\sin \psi\rangle=n_{2}^{-1} \sum_{y} y n_{2 y}
$$

where $n_{2 y}$ is the number of solute molecules that assume the disorientation index $y$, with $n_{2}=\sum_{y} n_{2 y} \omega_{y}$ is the a priori probability for the orientation index $y$. $\omega_{1}$ denotes the probability for perfect order. The first six terms in eq 2 follow from the improved lattice treatment of Flory and Ronca. ${ }^{4}$ The last term is due to the flow potential. $G$ is related to flow by

$$
G=C \dot{\gamma} b^{2} /(k T / \xi)
$$

where $\dot{\gamma}$ denotes the velocity gradient and $b$ is the size of a lattice cell which is also identical with the length of a rod segment. $k T / \xi$ is the diffusion coefficient as defined previously. ${ }_{13}^{13,14}$ The constant $C$ equals $1 / 8$ for elongational flow and $1 / 6$ for pure shear flow.

The orientation distribution function $f=n_{2 y} / n_{2}$ is obtained by differentiating eq 2 with respect to $n_{2 y}$ and equating to zero. Thus,

$$
f=\frac{n_{2 y}}{n_{2}}=f_{1}^{-1} \sin \psi \exp \left\{-\left(\alpha_{\text {lattice }}\right)\right\}
$$

where $\alpha_{\text {lattice }}=\alpha_{\mathrm{e}}+\alpha_{\mathrm{f}}$, with

$$
\begin{gathered}
\alpha_{\mathrm{e}}=-\frac{4}{\Pi} x \ln \left[1-v_{2}\left(1-\frac{4 f_{2}}{\Pi f_{1}}\right)\right] \sin \psi \\
\alpha_{\mathrm{f}}=\frac{G}{2}\left(x^{3}+3 x^{2}+2 x\right) \sin ^{2} \psi
\end{gathered}
$$

and

$f_{n}=\int_{0}^{\pi / 2} \sin ^{n} \psi \exp \left\{-\left(\alpha_{\text {lattice }}\right)\right\} \mathrm{d} \psi \quad(n=1,2, \ldots)$

The term $S$ represents the average orientation or the order parameter defined as

$$
S \equiv 1-\frac{3}{2}\left\langle\sin ^{2} \psi\right\rangle=1-\frac{3}{2} \frac{f_{3}}{f_{1}}
$$

\section{The Doi Theory and Comparison with the Lattice} Treatment

Doi's treatment of solutions of rodlike particles ${ }^{17}$ is based on the solution of the generalized form of the kinetic equation, i.e.,

$$
\partial f / \partial t=\nabla_{\mathrm{u}} \cdot \bar{D}_{\mathrm{r}}\left(\nabla_{\mathrm{u}} f+f \nabla_{\mathrm{u}} \alpha_{\mathrm{Doi}}\right)+\nabla_{\mathrm{u}} \cdot \mathbf{u} f
$$

Here $\mathbf{u}$ is the unit vector along the rod, $\bar{D}_{\mathrm{r}}$ is the overall rotational diffusion coefficient, and $\nabla_{\mathrm{u}}$ is the gradient operator with respect to $\mathbf{u}$. $f$ is the orientational distribution function, expressed in terms of the mean-field potential $k T \alpha_{\text {Doi }}$ as ${ }^{21}$

$$
f=f_{1}^{-1} \sin \psi \exp \left(\alpha_{\mathrm{Doi}}\right)
$$

with

$$
f_{1}=\int_{0}^{\Pi / 2} \sin \psi \exp \left(\alpha_{\mathrm{Doi}}\right) \mathrm{d} \psi
$$

$\alpha_{\text {Doi }}$ reduces the following form for orientation along a given axis:

$$
\alpha_{\text {Doi }}=\frac{9}{4} U S \sin ^{2} \psi-\frac{3}{2} U S
$$

Here, $U$ is defined ${ }^{17}$ as a dimensionless parameter proportional to the concentration and the size of the rods, i.e.,

$$
U=\nu_{2} x v_{2}
$$

where $\nu_{2}$ is a numerical factor. It is clear that the second term in eq 14 , which is independent of $\psi$, will be eliminated in eq 12 , so it may be neglected so far as the distribution function is concerned. The mean field potential $k T \alpha_{\text {Doi }}$ given by eq 14 reflects Maier-Saupe ${ }^{20}$ type of interactions. 
The fundamental differences and similarities between the lattice treatment and Doi theory may now be identified by comparing eq 12-15 with eq 6-9. Expanding the logarithmic term in eq 7 and retaining the first term only we obtain

$$
\alpha_{e} \simeq \frac{4}{\Pi} \sin \psi\left(1-\frac{4}{\Pi}\langle\sin \psi\rangle\right) x v_{2}
$$

This approximation is satisfactory for relatively long rods $(x \gg 10)$ whose transitions occur at low concentrations.

On the other hand, substituting eq 15 into eq 14 and using eq 10 we obtain the $\psi$-dependent term of the Doi theory as

$$
\alpha_{\text {Doi }}=\frac{9}{4} \nu_{2} \sin ^{2} \psi\left(1-\frac{3}{2}\left\langle\sin ^{2} \psi\right\rangle\right) x v_{2}
$$

Equations 16 and 17 allow for the direct comparison of the two theories. Linear dependence of the two on $x$ and $v_{2}$ is an important common feature. The remaining terms, although not identical, exhibit similar dependence on $\psi$ in the interval $0<\psi<\Pi / 2$. It has been pointed out by Flory and Ronca ${ }^{5}$ that the precise form of the orientational distribution function is of secondary importance. Calculations to be reported below indeed show that the use of the potential of eq 17 instead of eq 16 and proper rescaling of $\nu_{2}$ lead to results similar to those obtained by the lattice treatment.

The effect of the flow field is introduced into the potential of the lattice treatment through eq 8 .

As the flow rates increase, the term given by eq 8 starts to gain importance relative to the static term in the potential. In the present study we assume that while the form of the stress constitutive equation given by Doi remains the same, the effective potential becomes modified by the addition of the flow term.

The governing equations resulting from these modifications may be summarized as follows.

(i) The orientational distribution function, the meanfield potential, and the order parameter: The orientational distribution function is given by the equation

$$
f=f_{1}^{-1} \sin \psi \exp (-\alpha)
$$

where $f_{1}$ follows from the general expression

$$
f_{n}=\int_{0}^{\pi / 2} \sin ^{n} \psi \exp (-\alpha) \mathrm{d} \psi
$$

The symbol $\alpha$ in eq 18 and 19 is defined as $\alpha=\alpha_{\mathrm{f}}+\alpha_{\text {Doi }}$ which leads to

$$
\alpha=\left\{\frac{G}{2}\left(x^{3}+3 x^{2}+2 x\right)+\nu S x v_{2}\right\} \sin ^{2} \psi
$$

Here $G$ retains its previous definition, $\nu=9 \nu_{2} / 4$, and the order parameter is calculated from eq 10.

(ii) Relationship of viscosity to orientation: For simple shear flow, the viscosity $\eta$ is given by the Doi theory as $\eta=$

$$
\eta_{0} x^{6} v_{2}^{3}(1-S)^{4}(1+S)^{2}(1+2 S)(1+3 S / 2)(1+S / 2)^{-2}
$$

where $\eta_{0}$ is a phenomenological front factor.

Equations 18-21 may be used to obtain relations between $S, G, v_{2}$, and $\eta$. The order parameter $S$ given by eq 10 has to be evaluated in a self-consistent manner by using the potential of eq 20 which also contains $S$. In general few iterations are sufficient to obtain convergence.

Equation 21 is only valid in the linear regime. It means that this theory will be able to give quantitative predictions up to the beginning of the shear-thinning region.

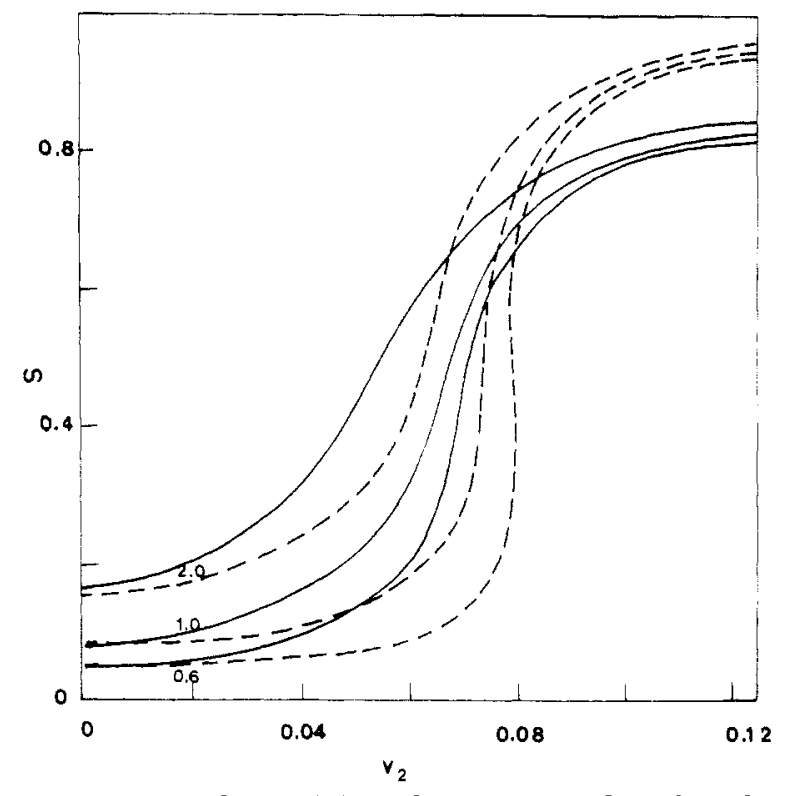

Figure 1. Dependence of the order parameter $S$ on the volume fraction $v_{2}$ of rodlike polymers. Solid curves are calculated for different flow parameters, $G$, by the present treatment (eq 18-20), with $\nu=0.9$ and $x=100$. The corresponding curves obtained ${ }^{14}$ by the lattice treatment are shown by the dashed lines.

\section{Numerical Calculations and Comparison with Experimental Data}

In order to compare the theory presented by eq 18-21 with experimental data, three parameters $G, \nu$, and $\eta_{0}$ have to be specified. They are not arbitrary. It is seen from eq 5 that $G$ is a known function of the type of flow, flow rate, segment size, and the solute diffusion coefficient. The second parameter reflects the characteristics of a given polymer-solvent system. At vanishingly small shear rates it is the only parameter that determines the complete phase transition characteristics of the system. In fact, suitable choice of $\nu$ leads to the appearance of a new highly anisotropic phase at the critical concentration $v^{*}$ predicted by eq $1 . \eta_{0}$ is a scaling factor required to match the experimentally observed viscosity values with those obtained by theory.

Results of calculations of $S$ for various values of $G$ are presented in Figure 1 as a function of volume fraction of rods. Calculations are performed for an axial ratio of 100 . Solid lines represent results obtained by using the potential given by eq 20. $v$ in chosen as 0.9 . This value is seen to lead to values of $v^{*}$ that are in agreement with previous calculations ${ }^{3,4,14}$ and experimental data of Hermans. ${ }^{6}$ The curves exhibit all features observed in real systems. At low values of $v_{2}, S$ is somewhat sensitive to variations in $G$. There is an interval of $v_{2}$ (approximately between 0.05 and 0.08 ) where the order parameter increases sharply with $v_{2}$. At higher concentrations, the curves obtained for different values of $G$ converge to the same $S$. The rapidly increasing part of the curves corresponds to the onset of the increase in viscosity observed experimentally as shown in the following. The dotted curves are results obtained by the lattice theory ${ }^{14}$ for $x=100$. There is no adjustable parameter in the lattice theory. Similarity of the two sets of curves both in shape and in location along the $y_{2}$ axis results from the fundamental agreement between the lattice and the Doi theories in this concentration range. The only free parameter to bring the two results into close proximity is the parameter $\nu$ of the Doi theory.

The relationship between steady shear viscosity and concentration is presented by the curves in Figure 3. Calculations are performed with the same parameters as 

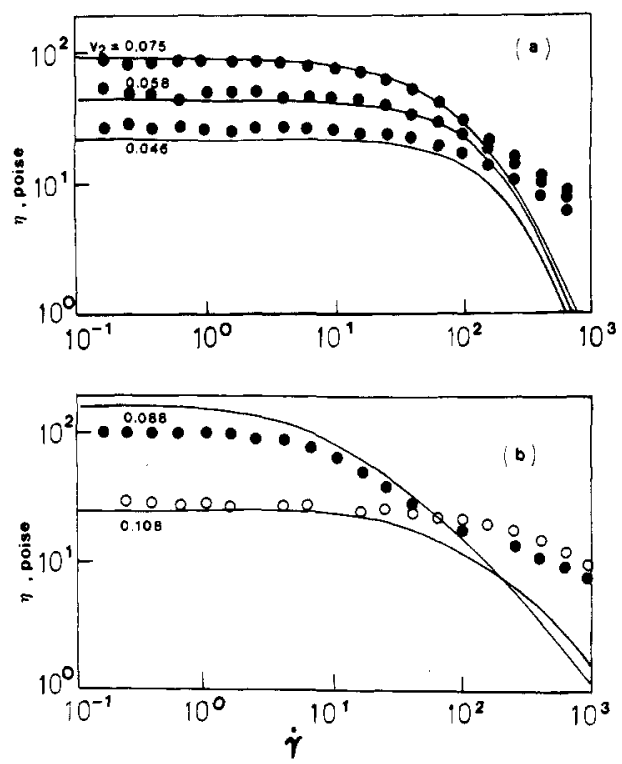

Figure 2. Dependence of viscosity on shear rate for various concentrations. Volume fractions $v_{2}$ are indicated on each curve. Circles represent data from experiments of Kiss and Porter. ${ }^{22}$ The curves are calculated by the present theory (eq 18-21) for $x=$ $67, \nu=1.1$, and $\eta_{0}=2.2 \times 10^{-6}$ and the shear rate is $\dot{\gamma}=2.8 \times$ $10^{7} \mathrm{G}$.

Figure 2. Filled and empty circles represent results of steady shear experiments of Kiss and Porter ${ }^{22}$ on solutions of PBLG in $m$-cresol with $x=67$. The weight fractions $c$ reported in their work have been converted to volume fractions by using $c=1.3 v_{2}$. The data for the uppermost curve which were obtained ${ }^{22}$ for zero-shear rate are approximated by taking $\dot{\gamma}=1 \mathrm{~s}^{-1}$. This is a plausible approximation since in the flow regime with $\dot{\gamma}<1 \mathrm{~s}^{-1}$, the viscosity values are insensitive to changes in $\dot{\gamma}$ but are mainly dominated by the static (equilibrium) contribution $\alpha_{\text {Doi }}$ to $\alpha$. The locations and magnitudes of the experimentally observed peaks are well represented by the theory. Although this theory cannot give quantitative information in the shear-thinning region, it agrees qualitatively with the experiments. That the peak should vanish for high shear rates is predicted by the lowest line. The shift of the peaks to smaller concentrations with increasing shear rate is also predicted by the theory. This observation has also been discussed by Doi and Edwards ${ }^{23}$ in relation to nonlinear viscoelasticity in nematics. Comparison of the reported values of $\dot{\gamma}$ with the values of $G$ required for agreement with Kiss and Porter's data show that

$$
G=\dot{\gamma} / 2.8 \times 10^{7}
$$

where $\dot{\gamma}$ is in reciprocal seconds.

Taking $b=15.6 \AA$ as deduced from X-ray determination of the unit cell ${ }^{24}$ and $c=1 / 6$ in eq 5 and comparing with eq 22 lead to a value of about $10^{-7} \mathrm{~cm}^{2} / \mathrm{s}$ for the diffusion coefficient, $k T / \xi$. It should be pointed out here that the state of flow in cone-and-plate experiments of Kiss and Porter is simple shear whereas the factor of $1 / 6$ in eq 5 is obtained for pure shear, where the vorticity term is zero. The effect of this difference on viscosity is expected to be inconsequential at lower shear rates (see sequel). The dashed portion of the curve for $\dot{\gamma}=1$ corresponds to the biphasic region. The order parameter and viscosity, obtained from eq 10 and 21, respectively, are double-valued when the system is biphasic. Details of calculations that lead to the biphasic region in Figure 3 are discussed below. It is worth noting that no biphasic region is observed in the remaining curves of Figure 3 .

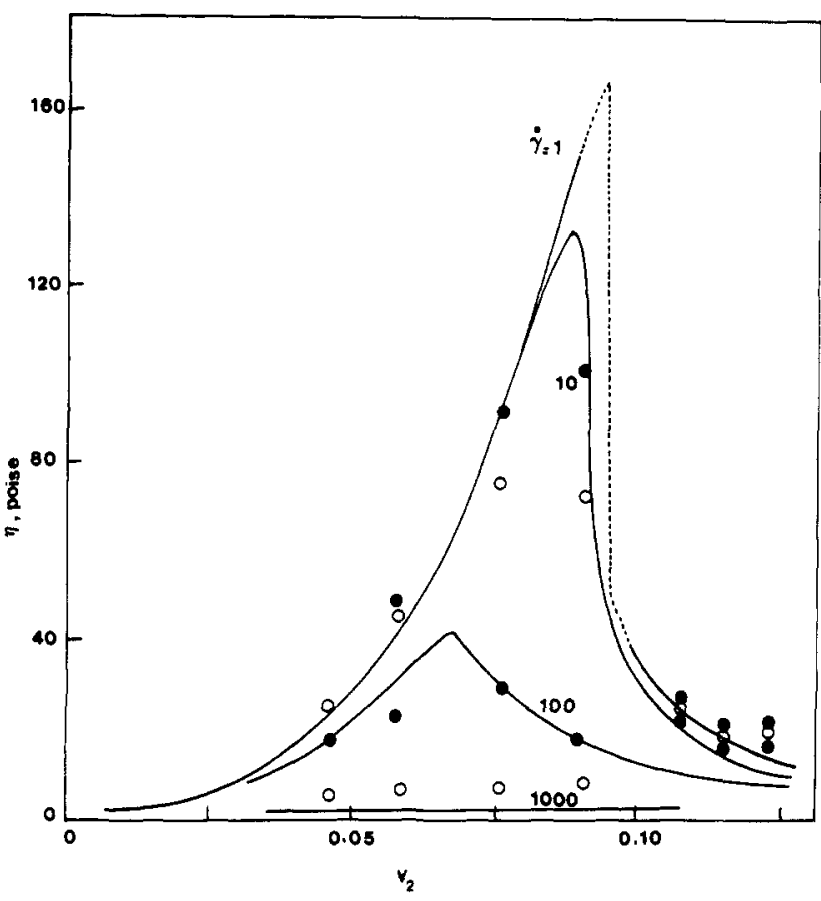

Figure 3. Change in the viscosity of the solution with volume fraction of rodlike polymers. The curves are calculated by the present treatment by using the same parameters as in Figure 2. Circles represent data from Kiss and Porter. ${ }^{22}$ The dashed portion of the uppermost curve represents the biphasic region. The shear rate is indicated on each curve.

The dependence of viscosity on shear rate $\dot{\gamma}$ is presented in Figure 2 for various concentrations. Calculations are performed according to eq 18-21 for $x=67, \nu=1.1, \eta_{0}=$ $2.2 \times 10^{-6}$. Filled circles represent experimental data of Kiss and Porter of PBLG with ${ }^{25} x=67$. Percentages of concentrations expressed in volume fractions are shown on each curve. The plateau region and the onset of the decline of viscosity with $\dot{\gamma}$ are predicted satisfactorily by the theory. The viscosity-shear rate curves cross each other if presented on the same graph which is also verified by the theory. The failure of the theory at high shear rates is due to the use of eq 21, only valid in the linear regime.

Predictions of the theory are compared in Figure 4 with results of viscosity experiments on PBLG by Hermans. ${ }^{6}$ The ordinate represents the viscosity. The abscissa is the volume fraction of rods. Results for PBLG in $m$-cresol, with molecular weight $342000(x=150)$, are shown by filled circles. Data for molecular weight $220000(x=97)$ are given by the empty circles. Parameters used in the theory are $\nu=0.8$ and $\eta_{0}=0.66 \times 10^{-6}$. Differences in $\eta_{0}$ for Kiss and Porter and Hermans data may be attributed to polydispersity effects. In Figure 4, only two sets of experiments are compared with theory. A third set with $x=120$ is not included because the peak value of $\eta$ for $x$ $=120$ is larger than those for $x=97$ and 150 . The predictions of the theory indicate, however, that the peak viscosity should be monotone, strictly increasing with rod length.

The order parameter and viscosity in and around the biphasic region are shown in more detail in Figures 5-7. The biphasic region is the region in which $S$ and $\eta$ are double-valued. The concentrations $v^{*}$ and $v_{2}{ }^{A}$ defining the boundary depend on the flow parameter, $G$. In Figure 5 this boundary is shown for a system with $x=67, \nu=1.1$. The flow parameter $G$ is plotted against the volume fraction of rodlike polymers. The area bounded by the curve is the biphasic region. Outside this boundary, the system is single phase, with different degrees of anisotropy 


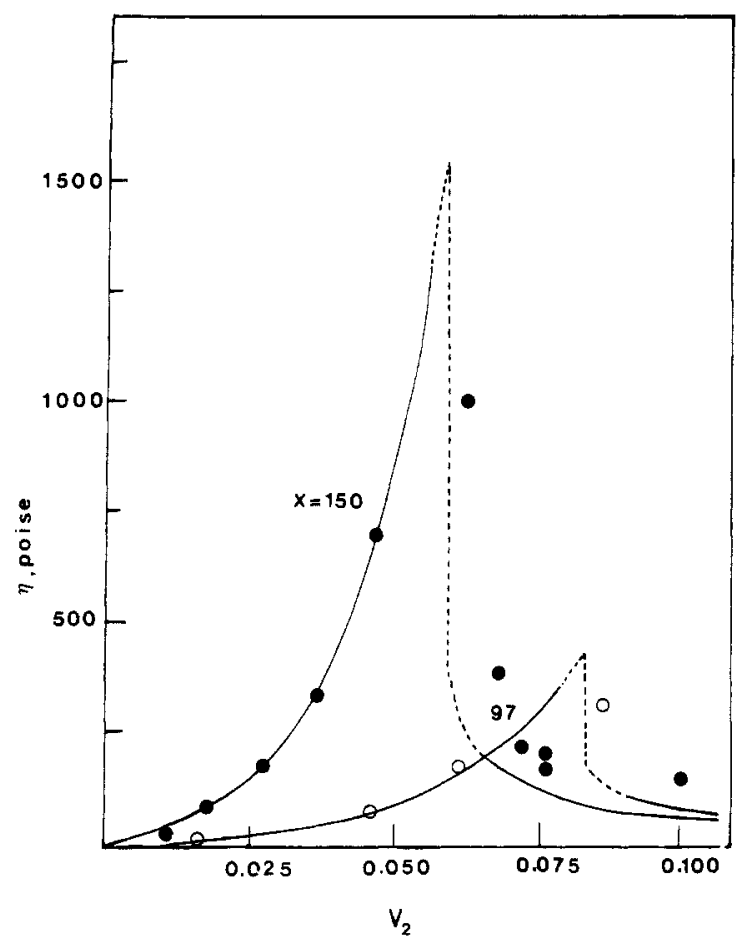

Figure 4. Change in viscosity with concentration for two axial ratios $x$, shown on each curve. Curves are calculated for zero shear rate, by using the parameters $\nu=0.8$ and $\eta_{0}=0.66 \times 10^{-6}$. Circles represent experimental data from the work of Hermans. ${ }^{6}$

depending on the value of $G$ and $v_{2}$. The parameters used in obtaining the boundary of the biphasic region of Figure 5 correspond to those used to reproduce the data of Kiss and Porter shown in Figure 2. It is thus concluded from Figure 2 that the system used by Kiss and Porter enters the biphasic regime only for the curve with $\dot{\gamma}=1$, while the curves for $\dot{\gamma}=10$ and 100 correspond to a single anisotropic phase with degree of anisotropy increasing with $\dot{\gamma}$ and $v_{2}$.

The relationship of the order parameter to concentration is shown in Figure 6. The two vertical dashed lines separate the biphasic region from those of low anisotropy on the left and those of high anisotropy on the right.

The order parameter in the low-anisotropy region is very close to zero; i.e., the system is almost isotropic. Thus this phase may be referred to as the isotropic phase to a good approximation. Values of $\eta$ calculated around and in the biphasic region are given in Figure 7. The viscosity of the disordered, isotropic phase is considerably higher than that of the highly anisotropic phase. The dashed curve represents the mean viscosity of the biphasic system obtained by weighting the viscosities of the two phases with their respective volume fractions in the mixture. The dotdashed curve is drawn by assuming a phase inversion that occurs at the mean of $v^{*}{ }_{2}$ and $v_{2}{ }^{A}$. The viscosity of the heterogeneous system is assumed to be determined by that of the isotropic phase until phase inversion after which the situation is reversed; i.e., the viscosity of the anisotropic phase dominates. The dashed portion of the uppermost curve in Figure 3 corresponds to this dot-dashed curve.

\section{Discussion and Conclusions}

Modification of the Doi theory by introducing a flow term into the effective potential of lyotropic liquid-crystalline systems leads to results which are in quantitative agreement with various aspects of experimental data over a wide range of flow rates. Several remarks follow from the present treatment.
The location of the maxima of viscosity is adjusted by the parameter $\nu . \nu$ was found to be in the range $0.8-1.1$ for the molecular weights we used. It is possible to calculate $\nu$ from the equilibrium properties of the solution. At the concentration $\nu^{*}$, when the phase separation occurs, Doi theory predicts that $\nu=3 \Pi / 4 x \nu_{2}^{*}$. This gives $\nu$ in the range $0.6-0.8$, which is very close to the values found by fitting the experimental data with the theory.

The experimentally observed shifting of the viscosity peaks to lower concentrations as the flow rate increases is predicted by the theory.

At low shear rates the increasing parts of the viscosity versus concentration curves almost coincide with experimental data as seen in Figures 3 and 4.

The theory predicts the Newtonian plateau preceding the decrease of $\eta$ with $\dot{\gamma}$, in satisfactory agreement with experimental findings as shown in Figure 2 for different values of $\nu_{2}$. As stated above, the theory fails in the shear-thinning region due to the use of eq 21 .

In addition to the use of eq 21 , the failure of the theory at high shear rates may be due to the fact that the theory is developed for pure shear whereas the experimental data are obtained under simple shear conditions. The two differ by the vorticity component which is present in the latter. The effect of the vorticity component is expected to be insignificant at rates which are much lower than the rotational diffusion of the rods. Satisfactory agreement of the present formulation with experimental data at low shear rates is an indication along this direction. However, a conclusive understanding of the differences between pure and simple shear flows in relation to molecular phenomena awaits further experimental work.

The strong dependence of the viscosity expression on rod length invites attention to effects that may arise from polydispersity and nonrigidity. Different values of $\eta_{0}$ required to describe the experiments of Hermans and Kiss and Porter may be ascribed to effects of polydispersity. The effects of nonrigidity of rods may be equally important as those of polydispersity. Comparison of a set of data ${ }^{9}$ from solutions of poly(benzylglutamate) containing $50 \%$ D peptide units and $50 \% \mathrm{~L}$ units leads to results incompatible with those for solutions containing $100 \% \mathrm{~L}$ units. Application of the present theory to solutions of HPC, for example, should be made with care inasmuch as this system is not a good example of a rigid polymer, and its degree of substitution is not regular. ${ }^{26,27}$

Although an estimate of the value of $\eta_{0}$ may be made by employing the molecular theory of Doi and Edwards, we presently do not pursue it due to the fact that while the Doi-Edwards theory is qualitatively correct, the predicted magnitudes of its parameters do not agree with experimental data. ${ }^{28,29}$ One indication along these lines is observed in the work of Zero and Pecora ${ }^{28}$ where the value of the rotational diffusion coefficient of PBLG is obtained to be 2 orders of magnitude higher than that predicted by the Doi-Edwards theory.

The boundary of the biphasic region is similar in shape to that of the lattice treatment. ${ }^{14}$ However, the biphasic gap under flow is rather narrow in contrast to the predictions of lattice theory, but in agreement with Doi's static theory of phase transition. However, the disagreement between the two theories as to the width of the biphasic gap is inconsequential in determining rheological properties. In fact as pointed out previously, ${ }^{11}$ the coexisting phases are distinguished more markedly by their degree of ordering than by the difference in their concentrations.

In the biphasic regime both the order parameter and consequently the viscosity are double-valued. The relative 


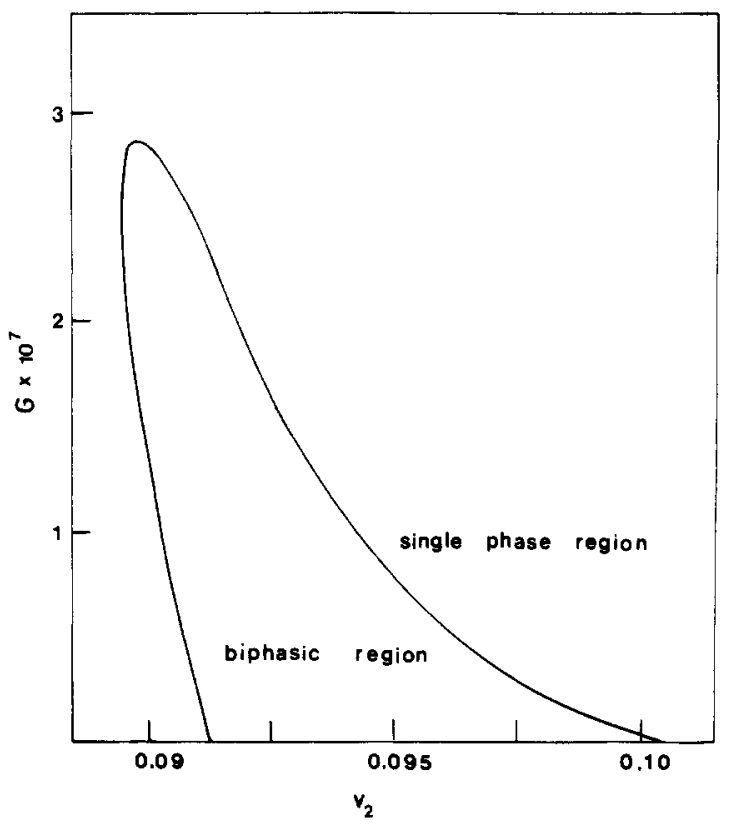

Figure 5. Biphasic region in solutions of rodlike polymers under flow for $x=67$ and $\nu=1.1$. The region bounded by the curve represents the biphasic system with double-valued order parameter $S$. In the region outside the curve, the system is single-phased with degree of anisotropy increasing with $G$ and $v_{2}$.

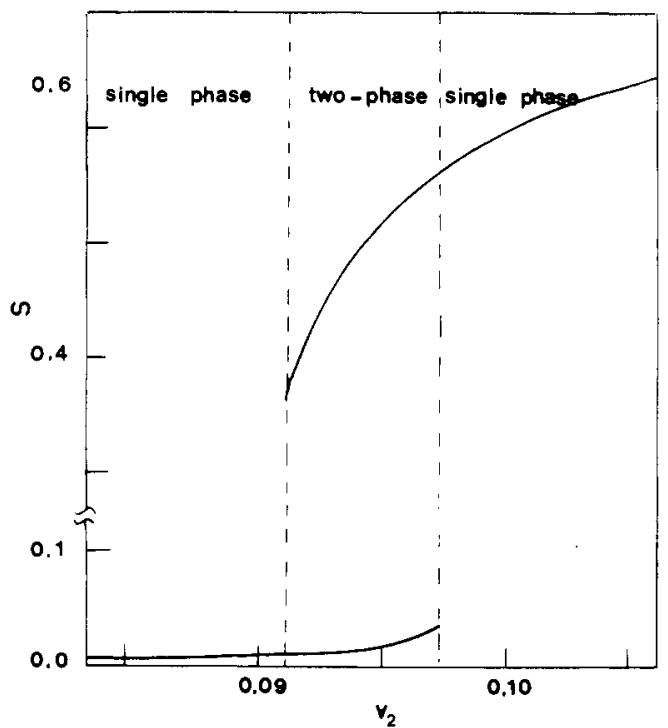

Figure 6. Variation of the order parameter $S$ with volume fraction $v_{2}$ of rods in and about the biphasic region, limited by the vertical dashed lines. The same parameters as in Figures 2 and 3 are used with $\dot{\gamma}=1$. The lower curve corresponds to the weakly ordered almost isotropic phase. The upper one exhibits the behavior of the highly anisotropic phase. The order parameter is doublevalued in the biphasic region.

contributions of the two phases to the overall viscosity of the biphasic solution are not well defined at the present. It should be added that the present mean-field treatment is not expected to predict the phenomena in the biphasic region in the presence of flow. Possibility of dynamic instabilities would prevent the application of the present theory to the biphasic region. In view of these difficulties, the biphasic region in Figure 3 is presented by dotted lines, and the discussion pertaining to Figures 6 and 7 should be regarded only as exploratory.

Finally, it should be pointed out that at vanishingly low shear rates, most of the main-chain nematic polymers, thermotropic or in solution, do not present a newtonian plateau but have a power-law region. This is supposed to

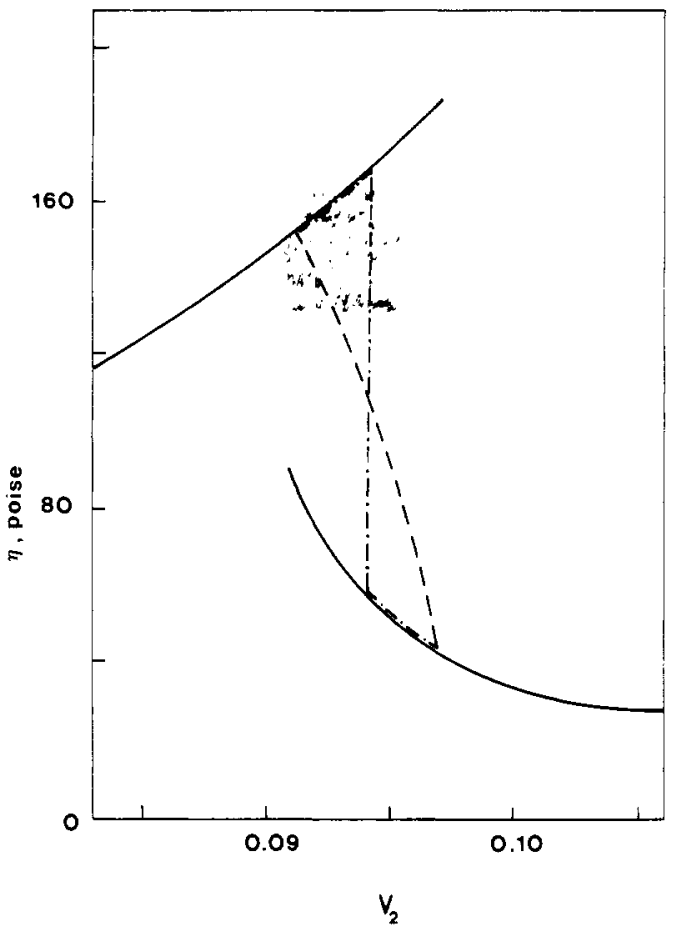

Figure 7. Variation of the viscosity with concentration in and about the biphasic region. The upper curve on the left stands for the phase with low anisotropy while the lower one is representative of the highly ordered anisotropic phase. In the intermediate biphasic region, the viscosities are double-valued. The dashed curve is drawn by weighting the viscosities in each phase by their respective volume fractions. The dot-dashed curve is drawn on the basis of a phase inversion occurring at the midpoint of the concentration interval.

be due to the presence of a certain structure ${ }^{30}$ present in the nematic phase. This problem is not addressed in the present theory.

Registry No. PBLG (homopolymer), 25014-27-1; PBLG (SRU), 25038-53-3.

\section{References and Notes}

(1) Onsager, L. Ann. N.Y. Acad. Sci. 1949, 51, 627.

(2) Isihara, A. J. Chem. Phys. 1951, 19, 1142.

(3) Flory, P. J. Proc. R. Soc. London A 1956, 234, 73

(4) Flory, P. J.; Ronca, G. Mol. Cryst. Liq. Cryst. 1979, 54, 289.

(5) Flory, P. J.; Ronca, G. Mol. Cryst. Lig. Cryst. 1979, 54, 310.

(6) Hermans, J. J. J. Colloid Sci. 1962, 17, 638.

(7) Nakajima, A.; Hayashi, T.; Ohmori, M. Biopolymers 1968, 6, 973.

(8) Wee, E. L.; Miller, W. G. Liquid Crystals and Ordered Fluids; Johnson, J. F., Porter, R. S., Eds.; Plenum: New York, 1978 Vol. 3, p 371.

(9) Kiss, G.; Porter, R. J. Polym. Sci., Polym. Symp. 1978, No. 65, 193.

(10) Aoki, H.; White, J. L.; Fellers, J. F. J. Appl. Polym. Sci. 1979, 23,2293 .

(11) Flory, P. J. Adv. Polym. Sci. 1984, 59, 1.

(12) Matheson, R. R. Macromolecules 1980, 13, 643.

(13) Marrucci, G.; Ciferri, A. J. Polym. Sci., Polym. Lett. Ed. 1977, 15,643 .

(14) Bahar, I.; Erman, B. J. Polym. Sci., Polym. Phys. Ed. 1986, 24, 1361.

(15) Doi, M. J. Phys. (Les Ulis, Fr.) 1975, 36, 607.

(16) Doi, M.; Edwards, S. F. J. Chem. Soc., Faraday Trans. 2 1978, 74,$560 ; 1978,74,918$.

(17) Doi, M. J. Polym. Sci., Polym. Phys. Ed. 1981, 19, 229.

(18) Metzner, A. B.; Prilutski, G. M. J. Rheol. (N.Y.) 1986, 30, 661.

(19) Doraiswamy, D.; Metzner, A. B. Rheol. Acta 1986, 25, 580

(20) Maier, W.; Saupe, A. Z. Naturforsch., A: Astrophys., Phys. Phys. Chem. 1958, 13, 564.

(21) The term $\sin \psi$ in eq 12 , which is not present in the expression given by Doi, results from representation in terms of the angle 
$\psi$ that a rod makes with the given direction of orientation.

(22) Kiss, G.; Porter, R. S. J. Polym. Sci., Polym. Phys. Ed. 1980, 18,361 .

(23) Doi, M.; Edwards, S. F. The Theory of Polymer Dynamics; Clarendon: Oxford, 1986.

(24) Sasaki, S.; Naka, M.; Uematsu, I. Polym. J. (Tokyo) 1982, 14, 465 .
(25) Values of $x$ reported in this work are taken from ref 11.

(26) Navard, P.; Haudin, J. M. J. Polym. Sci., Polym. Phys. Ed. $1986,24,189$.

(27) Navard, P. J. Polym. Sci., Polym. Phys. Ed. 1986, 24, 435.

(28) Zero, K. M.; Pecora, R. Macromolecules 1982, 15, 87.

(29) Keep, G. T.; Pecora, R. Macromolecules 1985, 18, 1167.

(30) Wissbrun, K. J. Rheol. (N.Y.) 1981, 25, 619.

\title{
(Carboxymethyl)cellulose with Xanthan Gum Like Rheology
}

\author{
D. J. Sikkema* and H. Janssen \\ Enka BV, Research Institute Arnhem, Velperweg 76, Arnhem, Netherlands. \\ Received December 4, 1987
}

\begin{abstract}
By manipulation of the alkalinity during (carboxymethyl)cellulose (CMC) preparation-aiming at the formation of poorly substituted segments, in fact at a cellulose-co-CMC segmented block copolymer-CMCs can be made with solution rheograms closely resembling those of xanthan gum. Such results were obtained by reacting both in an aqueous isopropyl alcohol slurry and in a "dry" phase with use of a kneader. Some salt resistance can be achieved, although not on the level that it can with xanthan gum. The new products show a temperature dependence of solution viscosity comparable with that of xanthan gum but do not show the discontinuity at $60^{\circ} \mathrm{C}$ that is characteristic of xanthan gum. ${ }^{6}$
\end{abstract}

\section{Introduction}

The extreme pseudoplasticity of xanthan gum solutions has long served as both a yardstick of performance for other water-soluble polymers and as an unattainable ideal for semisynthetic cellulose derivatives. ${ }^{1}$ Earlier work investigating the effect of oligosaccharide substituents flexibly coupled to a CMC backbone in a random substitution pattern-presenting a "random analogue" of xanthan gum's highly regular cellulose-graft-oligosaccharide structure-failed to produce unusual rheologies, although a very high level of viscosity was attained, normalized to $1 \%$ cellulosic solutions. ${ }^{2}$

Literature reports ${ }^{3}$ that xanthan gum is organized into multistranded helical aggregates, forming fairly rigid, extremely long arrays, not unlike staple fiber yarn being built up from short fibers, inspired the approach described in this work. We aimed at the synthesis of CMC types that contain "cellulose blocks" consisting of segments of poorly substituted CMC, which would act as physical cross-links in the solution, to produce multistranded CMC colloidal fibers. Such a conjugation of CMC molecules via cellulose-like segments would tend to align the relatively rigid ${ }^{4}$ cellulose derivative into bundles with an effective length far exceeding the individual molecular dimensions. This would lead to a strong increase of shear-thinning behavior due to increased orientability of such colloidal fibers, compared with the isolated molecules.

\section{Results}

Our synthesis follows the same general outline as the preparation of regular CMC: Alkalization to open up the cellulose structure as well as to generate high nucleophilic reactivity, followed by reaction with chloroacetic acid or its derivatives.

We open the cellulose structure by using an amount of alkali in the usual range, normally regarded sufficient to destroy all crystallinity: then, just prior to the start of the carboxymethylation reaction, we adust the alkalinity to a much lower range, inviting incipient recrystallization. The microcrystals locally protect the cellulose from substitution reactions; the unsubstituted regions furnish the poorly substituted "cellulose blocks" in the block-co-
Table I

CMC-Cellulose Segmented Block Copolymers from Reaction of Linters Cellulose ${ }^{a}$

\begin{tabular}{|c|c|c|c|c|c|}
\hline entry & $\begin{array}{l}\text { MCA, } \\
\text { equiv }\end{array}$ & $\begin{array}{l}\text { MCA-Na, } \\
\text { equiv }\end{array}$ & $\begin{array}{c}\eta_{1 \mathrm{~s}^{-1}} \\
\mathrm{mPa} \cdot \mathrm{s}\end{array}$ & $\begin{array}{c}\eta_{20 \mathrm{~s}^{-1}} \\
\mathrm{mPa} \cdot \mathrm{s}\end{array}$ & $100 B$ \\
\hline 1 & 0 & 1.0 & 1250 & 700 & 23 \\
\hline 2 & 0.3 & 0.7 & 1750 & 880 & 35 \\
\hline 3 & 0.5 & 0.5 & 1920 & 880 & 35 \\
\hline 4 & 0.7 & 0.3 & 5920 & 1600 & 47 \\
\hline 5 & 0.7 & 0.3 & 6750 & 1980 & $47^{b}$ \\
\hline $6^{*}$ & 0.7 & 0.3 & 30200 & 4700 & 68 \\
\hline $7^{*}$ & 0.7 & 0.3 & 23600 & 4200 & $63^{c}$ \\
\hline $7 *$ & $0.75 \mathrm{w}$ & t \% soln & 13300 & 2460 & 61 \\
\hline $7^{*}$ & $0.50 \mathrm{w}$ & t \% soln & 3800 & 1100 & 52 \\
\hline 8 & 0 & 1.4 & 1810 & 650 & 40 \\
\hline 9 & 0.1 & 1.3 & 1410 & 690 & $35^{d}$ \\
\hline 10 & 0.2 & 1.2 & 2800 & 1060 & 22 \\
\hline 11 & 0.4 & 1.0 & insol & & \\
\hline various & $>0.4$ & $<1.0$ & insol & & \\
\hline $1.0 \%$ xanthan gum & & & 2800 & & 70 \\
\hline $\mathrm{AZC} \mathrm{HS} 600^{e}$ & & & 149 & 77 & 22 \\
\hline
\end{tabular}

${ }^{a} \mathrm{ER} 4500$; runs marked with * ER 8500, milled in the Pulverisette, with 1.5 equiv of $\mathrm{NaOH}$ (equivalents always relative to anhydroglucose) in $87 \%$ aqueous isopropyl alcohol and with various amounts of chloroacetic acid and sodium chloroacetate. Viscosity data for $1 \%$ solids unless indicated otherwise in all tables. Pseudoplasticity characterized by the factor $B(\log \tau=A-B \log \dot{\gamma})$; the mean value of $B$ in the slightly curved plots between 10 and $100 \mathrm{~s}^{-1}$ shear rate is reported, cf. ref 6 . ${ }^{b}$ Run 5 checks the reproducibility of run $4,{ }^{\circ}$ Run 7 reproduces run $6 .{ }^{d}$ Observe salt effect. ${ }^{e} \mathrm{Cf}$. Table V.

polymer-like product. Chloroacetic acid can be used for the partial neutralization if the temperature of the mixture is sufficiently low to preclude intervention of the etherification reaction in the neutralization step-i.e., below room temperature.

In searching for a good procedure to achieve soluble products containing segments of poorly substituted cellulose (Table I) we observed a pronounced salt effect on the collapse of the alkali cellulose structure (bottom part of Table I). Note that salt is expected to exert its effect in a less homogeneous and reproducible manner than $\mathrm{pH}$ control should, because of slow salt diffusion compared to the rate of proton exchange. Thus, in striving for a higher overall degree of substitution (DS), the use of increased 